\title{
Maximum super angle optimization method for array antenna pattern synthesis
}

\author{
Wu, Ji; Roederer, A. G
}

Published in:

Antennas and Propagation Society International Symposium

Link to article, DOI:

10.1109/APS.1991.175187

Publication date:

1991

Document Version

Publisher's PDF, also known as Version of record

Link back to DTU Orbit

Citation $(A P A)$ :

Wu, J., \& Roederer, A. G. (1991). Maximum super angle optimization method for array antenna pattern synthesis. In Antennas and Propagation Society International Symposium (Vol. Volume 3, pp. 1712-1715). IEEE. https://doi.org/10.1109/APS.1991.175187

\section{General rights}

Copyright and moral rights for the publications made accessible in the public portal are retained by the authors and/or other copyright owners and it is a condition of accessing publications that users recognise and abide by the legal requirements associated with these rights.

- Users may download and print one copy of any publication from the public portal for the purpose of private study or research.

- You may not further distribute the material or use it for any profit-making activity or commercial gain

- You may freely distribute the URL identifying the publication in the public portal 
MAXIMN SUPER ANGLE OPTIMIZATION METHOD FOR ARRAY ANTENNA PATTERN SYNTHESIS

\author{
Ji Wu+* A.G. Roederer ${ }^{++}$ \\ +Technical University of Denmark \\ Building 348, EMI, DK-2800, Lyngby, Denmark \\ ++ ESAESTEC, Postbus 299, 2200 AG, Noordwijk \\ The Netherlands
}

\begin{abstract}
Different optimization criterias related to antenna pattern synthesis are discussed. Based on the maximin criteria and vector space representation, a simple and efficient optimization method is presented for array and array fed reflector power pattern synthesis.
\end{abstract}

Least Square, Minimax and Maximin

Dealing with non-linear optimization problems, different objective functions and design error criterias may lead to very different solutions. Least square criteria [1][2] gives the smallest error in a statistical point of view. Which means that although the error is the smallest in general, at some sampling points they may be still large. Minimax is a better criteria [3][4] because it gives equally distributed errors almost everywhere, therefore the largest error is less than for the least square solution. The problem of both criterias is that because they all deal with the error functions, the results do not yield optimum gain. In order to get the optimum gain, an iterative procedure where the goal gain has to be increased progressively must be considered. Another problem for them is that they reduce both the positive and the negative errors of the designed pattern during the optimization.

Maximin is a criteria [5][6] which does not involve with the error function. It maximize the minimum gain in the main beam region which corresponds to working on the largest negative error all the time. In fact, the power given to the negative error comes from the positive errors but without any constraint to the optimization. At the end of the optimization, the pattern can be very well shaped following the desired one and the gain is optimized at the same time.

Basic Formulation in Vector Space

The following discussion will be limited to field magnitude (power) pattern synthesis by optimizing the complex element excitation coefficients. The array elements can have arbitrary element patterns but fixed positions. The composite field at the far field sampling points are

$$
E_{j}=\left\langle e_{j}, a^{*}\right\rangle=\sum_{i=1}^{n} e_{j i} a_{i} \quad(j=1, m)
$$

where $m$ is the number of far field sampling points, $n$ is the number of elements. $e_{j}(j=1, m)$ are the field vectors in n-dimensional Hilbert space. $e_{j i}$ is calculated by the analysis procedure of the array at the

$91 \mathrm{CH} 3036-1 / 0000-1712 \$ 1.0001991$ IEEE

1712 
jth sampling points from the ith array element only which is excited by a unit voltage wave with zero phase. $a$ is the excitation coefficient vector. Consider the field magnitude only, (1) is equivalent to:

$$
\begin{array}{ll}
\qquad\left|E_{j}\right|=<F_{j}, a^{*}>\quad(j=1, m) \\
\text { where } \quad F_{j}=e_{j}<e_{j}, a^{*}>^{*} /\left|<e_{j}, a^{*}>\right|
\end{array}
$$

$F_{j}$ is related to the current direction of $a$ and will be still called field vector in the following discussion. Considering the input power normalization, the unconstrained maximin optimization formula is then:

$$
\left.\max _{\mathbf{a}, \mathbf{F}_{\mathbf{j}} \in \mathrm{H}^{\mathbf{n}}}^{(\min }<\underset{1 \leq j \leq \mathrm{m}}{\mathbf{j}, \mathbf{a}^{*}}>/|| \mathbf{a} \|\right)
$$

Transformed Objective Function

Equation (4) maximizes the minimum projection length of $F_{j}(j=1, m)$ on a. The result of this optimization is that many field vectors which are called the active set will have the same projection length on $a$. The extremities of those vectors define a super plane. The extremities of the other vectors which give higher gain respect to the minimum will be above the plane on the other side of the origin. Therefore the necessary condition of an optimum solution has been derived as in [7]: an optimum excitation vector $a$ is in the direction of the normal of the super plane. Because only the direction of the excitation vector $\mathbf{a}$ is of interest, $\mathbf{a}$ un-normalized excitation vector $\mathbf{c}$ can be introduced. If the extremity of $\mathrm{c}$ is in the super plane at the end of the optimiza-tion, the above condition can be rewritten as:

$$
\begin{array}{ll}
c \perp\left(c-F_{j}\right) & (j=1, k) \\
\text { or } \quad \text { ang }\left\langle c,\left(c-F_{j}\right)\right\rangle \geq \pi / 2 . & (j=1, m)
\end{array}
$$

Where $\mathrm{k}$ is the number of active field vectors. The angle between $\mathrm{c}$ and $c-F_{j}$ is called the field angle. According to (6) the maximin optimization formula can be transformed to

$$
\max _{\mathbf{c}, \mathbf{F}_{\mathrm{j}}, \mathrm{H}^{\mathrm{n}}}\left(\min _{1 \leq \mathrm{j} \leq \mathrm{m}} \text { ang }<\mathbf{c},\left(\mathbf{c}-\mathbf{F}_{\mathrm{j}}\right)>\right\}
$$

\section{Super Angle Optimization Algorithm}

Super angle method is an algorithm specially adapted to Equation (7) in another publication [5]. It has been improved recently both in formulation and in computational time. A brief description of the algorithm follows:

1.Initialization: Set initial parameters, weigh the main beam and sidelobe field vectors with their specifications values and set initial excitation vector $c$ with its norm equal to the smallest norm of the main beam field vectors and its direction pointing to the field vector which is sampled in the centre of the main beam. 
2.Field angle search: Compute all the main beam field angles, select the field vector $F_{j s}$ which give the smallest angle and the biggest projection length $\mathrm{Pb}$ among those their projection lengths are less than the norm of $c$.

3.Perpendicular condition: If the smallest angle is less than $90^{\circ}$, reduce the norm of $\mathrm{c}$ to $\mathrm{Pb}$ and change both direction and the norm of $c$ towards to the direction of $F_{j s}$ in one step till the angle becomes to $90^{\circ}$, as show in Fig.1.

4.Sidelobe suppression: If there is sidelobe field vectors, compute all the sidelobe field angles, select the field vector $F_{j b}$ which give the biggest angle, if the biggest angle is greater than $90^{\circ}$, rotate c away from $F_{j b}$ in one step till the angle becomes to $90^{\circ}$ and keeping the same norm of $\mathbf{c}$, see Fig.2.

5.Result inspection: If the smallest field angle is equal or greater than $90^{\circ}$ and the biggest sidelobe error is within a pre-specified number, go to step 6 . Otherwise go back to step 2 .

6.Refine local maximum result: If the current minimum gain in the main beam is improved compared with the last run, increase $c$ by a factor which related to the improvement on the minimum gain, then go back to step 2. Otherwise turn to step 7.

7.Global maximum search: If the current minimum gain in the main beam is improved compare with the last global maximum search, the norm of $c$ is increased by a factor which ensures that when the program go back to step 2, c will rotate to another quadrant of the n-dimensional Hilbert space and keep the same relation as before with the field vector which gives the smallest field angle. Otherwise stop. The best result is $\mathbf{a}=\mathbf{c} /|\mathbf{c}| \mid$.

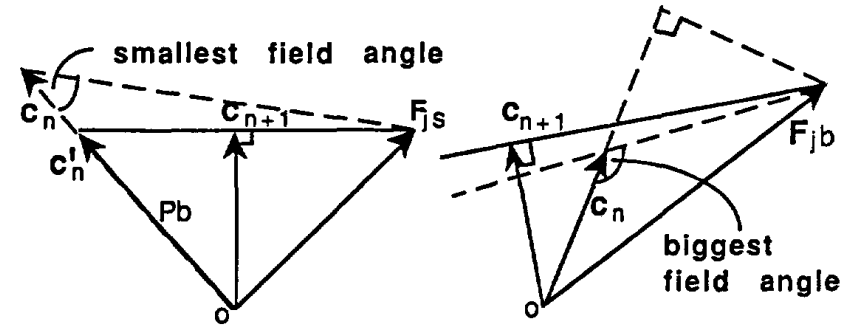

Fig.1

Fig.2

Examples

Fig. 3 shows a sector pattern synthesized by a 20 -element linear array. The $3 \mathrm{~dB}$ beamwidth of the main beam is $60^{\circ}$ and the sidelobes must be lower than -20dB respect to the main beam. Fig.4 is an example of a narrow, sector beam with very strict sidelobe specifications. The desired and synthesized patterns are shown in dashed and solid line respectively.

\section{Conclusions}


The maximin super angle method is a simple but fast and powerful method. Many array pattern parameters can be optimized under the same formulation. The transformed angle objective function (7) is specially adapted to the problem and has many advantages. First, it can be calculated directly from the field and excitation vectors without knowing the gradient and inverting a matrix. Secondly, a small change of field angle may correspond to a big change in the vector space, therefore it is efficient. Finally, the necessary and sufficient condition derived by the vector space representation [7] for the global maximum solution has been implemented.

\section{References}

[1] G.A. Deschamps and H.S. Cabayan, "Antenna synthesis and solution of inverse problems by regularization methods", IEEE Trans., AP-20, pp.268-274, May 1972

[2] J.R. Mautz and R.F. Harrington, "Computational methods for antenna pattern synthesis",IEEE Trans.,AP-23, p.507, July 1975

[3] H.S. Jacobsen and K. Madsen, "Synthesis of nonuniformly spaced arrays using general nonlinear minimax optimization method", IEEE Trans., AP-24, pp.501-506, July 1976

[4] C.A. Klein, "Design of shaped-beam antennas through minimax gain optimization", IEEE Trans., AP-32, pp.963-968, Sept. 1984

[5] J. Wu and A.G. Roederer, "The maximin optimization method for contoured-beam satellite antennas", ESA Journal, Vol.12, 1988

[6] J. Wu, "Maximin excitation coefficients optimization for array pattern synthesis", Report, No.R417, EMI, Technical University of Denmark, Feb. 1990

[7] J. Wu and A.G. Roederer, "Vector space representation of array antenna pattern synthesis problems", to be presented at AP-S Symposium, London, Canada, June 1991
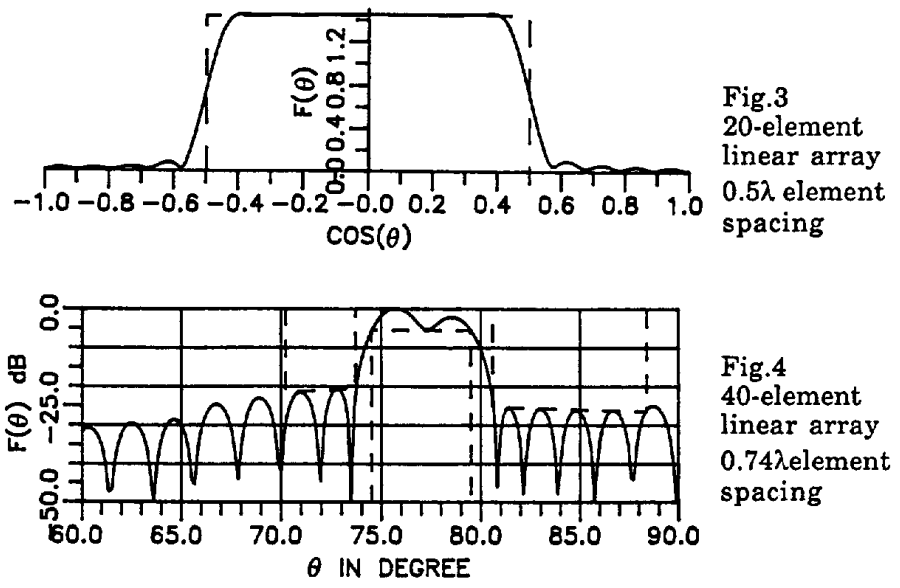

Fig.4

40-element

linear array

$0.74 \lambda$ element spacing 